\title{
Non-ergodicity of inviscid two-dimensional flow on a beta-plane and on the surface of a rotating sphere
}

Article

Published Version

Shepherd, T. G. (1987) Non-ergodicity of inviscid twodimensional flow on a beta-plane and on the surface of a rotating sphere. Journal of Fluid Mechanics, 184 (1). pp. 289302. ISSN 0022-1120 doi:

https://doi.org/10.1017/S0022112087002891 Available at https://centaur.reading.ac.uk/32991/

It is advisable to refer to the publisher's version if you intend to cite from the work. See Guidance on citing.

Published version at: http://dx.doi.org/10.1017/S0022112087002891

To link to this article DOI: http://dx.doi.org/10.1017/S0022112087002891

Publisher: Cambridge University Press

All outputs in CentAUR are protected by Intellectual Property Rights law, including copyright law. Copyright and IPR is retained by the creators or other copyright holders. Terms and conditions for use of this material are defined in the End User Agreement.

www.reading.ac.uk/centaur 
Central Archive at the University of Reading

Reading's research outputs online 


\title{
Non-ergodicity of inviscid two-dimensional flow on a beta-plane and on the surface of a rotating sphere
}

\author{
By THEODORE G. SHEPHERD \\ Department of Applied Mathematics and Theoretical Physics, University of Cambridge, \\ Silver Street, Cambridge CB3 9EW, UK
}

(Received 4 November 1986)

It is shown that, for a sufficiently large value of $\beta$, two-dimensional flow on a doubly-periodic beta-plane cannot be ergodic (phase-space filling) on the phase-space surface of constant energy and enstrophy. A corresponding result holds for flow on the surface of a rotating sphere, for a sufficiently rapid rotation rate $\Omega$. This implies that the higher-order, non-quadratic invariants are exerting a significant influence on the statistical evolution of the flow. The proof relies on the existence of a finite-amplitude Liapunov stability theorem for zonally symmetric basic states with a non-vanishing absolute-vorticity gradient. When the domain size is much larger than the size of a typical eddy, then a sufficient condition for non-ergodicity is that the wave steepness $\epsilon<1$, where $\epsilon=2 \sqrt{ } 2 Z / \beta U$ in the planar case and $\epsilon=2^{4} a^{\frac{b}{2}} Z^{2} / \Omega U^{\frac{\hbar}{2}}$ in the spherical case, and where $Z$ is the enstrophy, $U$ the r.m.s. velocity, and $a$ the radius of the sphere. This result may help to explain why numerical simulations of unforced beta-plane turbulence (in which $\epsilon$ decreases in time) seem to evolve into a non-ergodic regime at large scales.

\section{Introduction}

The governing equation of two-dimensional inviscid fluid flow is time reversible, in the sense that reversing the velocity field at a given instant will cause the system to 'run backward' to its initial configuration. Nevertheless, particular flow realizations invariably exhibit irreversible behaviour, namely turbulent cascades of energy to large scales and of enstrophy (mean-square vorticity) to small scales; that is, the time-reversible governing equation appears to possess an 'arrow of time'. This apparent paradox is, of course, a classical one whose resolution is well known (see Carnevale 1982; and in a more general context, Peierls 1979, §3.8).

In trying to understand the nonlinear behaviour of two-dimensional fluid flow, theoreticians have therefore frequently appealed to statistical approaches. Such approaches necessarily involve assumptions about the statistical evolution of a hypothetical ensemble of flow realizations. For example, by assuming nothing more than the broadening of spectral lines with time (e.g. Batchelor 1953, pp. 186-7), one can immediately deduce the ubiquitous spectral cascade tendencies of twodimensional turbulence, mentioned above, from the rigorous (inviscid) conservation of total energy and total enstrophy.

Underlying such statistical assumptions is a presumption of some sort of ergodic behaviour within the phase space of the problem. Ergodicity means that (almost all) phase-space trajectories fill out (in the sense of coming arbitrarily close to each point) the subset of phase space which is consistent with the relevant global invariants; its meaning therefore depends on which invariants are imposed. Compared with 
classical statistical-mechanical problems, where energy is the relevant invariant (e.g. Prigogine 1980, p. 33), the problem of inviscid two-dimensional flow is less straightforward because it has an infinity of global invariants (apart from the energy, and the linear and angular momenta, any functional of the vorticity), and it is unclear which ones to impose.

To make progress, it has commonly been hypothesized (e.g. Kraichnan 1975) that the statistical evolution of the flow is governed by the two quadratic invariants of energy and enstrophy. Ergodicity is then taken to mean that trajectories fill out the subset of phase space corresponding to fixed values of energy and enstrophy; this is the sense which concerns us here. Since energy and enstrophy reflect only the total scale of disturbance components, and not their orientation (stated otherwise, they are insensitive to the angular distribution of the vorticity spectrum), it follows that ergodicity in this sense must imply isotropic ensemble-mean flow statistics.

Two-dimensional flow on a beta-plane (or on the surface of a rotating sphere, to which the beta-plane is a local planar approximation) possesses the same quadratic invariants as does classical two-dimensional flow. This suggests that, if the dynamics is indeed ergodic on the phase-space surface of constant energy and enstrophy, then unforced beta-plane turbulence (Rhines 1975; Holloway \& Hendershott 1977) should, eventually, become statistically isotropic - this despite the anisotropy of the betaplane geometry. Numerical experiments do not tend to support this, however; rather, the simulated flows develop significant zonal anisotropy in the sense that $\left.\left\langle u^{2}\right\rangle\right\rangle\left\langle v^{2}\right\rangle(u$ being the zonal $(x-)$ velocity component and $v$ the meridional $(y-)$ component). But this numerical evidence does not rule out the possibility that the approach to statistical isotropy has simply been slowed down. The end-state of unforced beta-plane turbulence is therefore a matter of controversy (e.g. Salmon 1982 ; Bennett \& Haidvogel 1983; Holloway 1986).

The purpose of this note is to point out that, for a sufficiently large value of $\beta$, two-dimensional inviscid flow on a beta-plane is provably not ergodic in the sense described above. A corresponding result is also shown to hold for flow on the surface of a rotating sphere, for a sufficiently rapid rotation rate $\Omega$. The proof makes use of a finite-amplitude Liapunov stability theorem (4.1) (or (A 2)) for disturbances to a steady, zonally-symmetric basic state with a non-vanishing absolute-vorticity gradient. The idea is, for given energy and enstrophy, and given $\beta$ (or $\Omega$ ), to attempt to represent the flow as a stable basic state plus disturbance which (by the stability theorem) cannot evolve into a state for which the total flow is isotropic. If one can construct a set of such representations that is non-trivial, in the sense of having positive measure, then there exist zonally-anisotropic regions of phase space, embedded within the larger space of constant energy and enstrophy, from which trajectories cannot access the isotropic regions - and vice versa. It follows, ipso facto, that for such parameter values, at least, two-dimensional flow on a beta-plane (or on the surface of a rotating sphere) cannot be ergodic on the phase-space surface of constant energy and enstrophy.

The plan of the paper is as follows. The arguments for ergodicity (with respect to constant energy and enstrophy) for classical two-dimensional flow $(\beta=0)$ are reviewed in $\$ 2$. The case $\beta \neq 0$ is then discussed in $\$ 3$. The finite-amplitude generalized Rayleigh theorem (for the beta-plane geometry) is stated and proved in $\S 4$, and then applied in $\$ 5$ to show that, under certain conditions (specifically whenever, for given values of energy and enstrophy, and given $\beta$, it is possible to represent the flow as a basic flow and disturbance satisfying (5.9) and (5.11)), ergodic behaviour is impossible for two-dimensional flow on a beta-plane. In $\$ 6$ a condition 
on $\beta$ guaranteeing non-ergodic behaviour, $(6.7)$, is derived. The relevance of these findings to numerical simulations of unforced beta-plane turbulence is discussed in \$7. Corresponding results for the spherical case are treated in Appendix A.

\section{The role of higher-order invariants in two-dimensional turbulence}

As already mentioned, it has commonly been hypothesized (see Kraichnan (1975) for perhaps the clearest explicit statement) that the statistical dynamics of classical two-dimensional turbulence is controlled by the two quadratic invariants of energy and enstrophy, the higher-order invariants (representing material conservation of vorticity) having no constraining influence on the ensemble-mean behaviour. Kraichnan (1975, p. 167) argued for the hypothesis that, compared to the evolution one would obtain subject only to the constraints of constant total energy and enstrophy, 'the effect of detailed (i.e. material) vorticity invariance on evolution is to produce a fine-graining of the vorticity field'. Since this fine-graining becomes increasingly less visible to the large-scale dynamics, in the sense that, beyond a certain time, it is not contained in any truncated spectral representation of the flow, he further hypothesized that 'detailed constancy of the scalar (vorticity) amplitude is lost and the remaining isolating constants of motion are the integrals, over space, of the scalar amplitude and its square'.

It would certainly be convenient if this hypothesis were true, as nobody has been able, thus far, to find a way to incorporate the information contained in the higher-order invariants in a statistical methodology. Moreover, the validity of the hypothesis has important quantitative implications, insofar as most turbulence closure models, whether implicitly or explicitly, build in an assumption of precisely this sort by the way in which they treat high-order moments. Indeed, one can prove an ' $H$-theorem', and thus ergodicity (on the surface of constant energy and enstrophy), for $E D Q N M$ closure (Carnevale, Frisch \& Salmon 1981). Perhaps significantly, an $H$-theorem has not, however, yet been proven for the full governing equation of two-dimensional fluid flow.

What is the present evidence for the validity of Kraichnan's hypothesis? In the first place, there is the approach to absolute (statistical mechanical) equilibrium observed in inviscid truncated models. One may consider a finite-dimensional dynamical system, such as a large but finite number of Fourier modes, representing the large-scale motion in two-dimensional flow, together with an algorithm for the nonlinear interactions which preserves the total energy and enstrophy of the system (Kraichnan 1967). Invoking the assumption of ergodicity in the guise of maximizing a suitable measure of entropy subject to constant energy and enstrophy (e.g. Kraichnan 1967; Basdevant \& Sadourny 1975; Salmon, Holloway \& Hendershott 1976; Carnevale 1982), one obtains an absolute equilibrium solution. The robust approach of such a (truncated) system to its absolute equilibrium has been repeatedly verified numerically (e.g. Fox \& Orszag 1973; Basdevant \& Sadourny 1975; Carnevale 1982; Bennett \& Haidvogel 1983), and the ergodicity of the dynamics has been studied by Kells \& Orszag (1978). This evidence does not, however, relate directly to the ergodicity of the full (untruncated) system, as the truncated system fails to conserve the higher-order invariants related to material conservation of vorticity.

Of course it is well known in any case that the absolute equilibrium solutions are unphysical, in the sense that they do not converge as the number of modes is increased (indeed no state of absolute equilibrium exists at all for the full system). Nevertheless it is usually argued that their qualitative aspects are relevant to the behaviour of 
the full, infinite-dimensional system. Of particular importance is the fact that the absolute equilibrium spectra are isotropic; it has been suggested (Herring 1975) that this accounts for the tendency toward isotropy observed in numerical simulations of high-resolution, weakly-dissipative flow. Indeed this tendency toward isotropy may be considered a second piece of evidence in favour of the ergodic hypothesis, and one which need not be directly connected to inviscid truncated models.

A third piece of evidence for ergodicity is that provided by closure models. Again the evidence is inconclusive: the closure schemes make the ergodic hypothesis, in one form or another, and come up with predictions agreeing with direct simulation over a wide variety of conditions (Herring et al. 1974). However it has recently come to light that existing closure models, while producing accurate results for strongly-forced turbulence, do not work so well for flows which are weakly forced at small scales (Herring \& MeWilliams 1985). This failure is not particularly surprising, insofar as it is precisely these latter flows which develop intermittency and coherent structures (Babiano et al. 1984; MeWilliams 1984) in which the higher-order moments are significantly non-Gaussian. $\dagger$

An interesting claim regarding ergodicity is made by Thompson (1982). He first shows that, under certain (very strong) conditions on the properties of the equation of motion, one may deduce material conservation of vorticity (and hence of the infinity of higher-order invariants) solely from global conservation of enstrophy. Thompson then suggests that this apparent redundancy justifies the neglect of higher-order invariants in statistical theories. The validity of such an inference is certainly not obvious, however, because a great deal of dynamical information is contained in his conditions. In fact, were the inference true, then for consistency one should also neglect conservation of energy in statistical theories since it, too, is derivable (under Thompson's conditions) from enstrophy conservation. But whatever the implications of Thompson's result are, they are not applicable to two-dimensional flow on a beta-plane: his conditions are violated in that case by the presence of a dimensional constant and by the inherent anisotropy of the beta-plane geometry.

\section{Two-dimensional turbulence on a beta-plane}

Both classical two-dimensional flow, and two-dimensional flow on a beta-plane, are governed by conservation of absolute vorticity $P$ :

$$
\frac{\mathrm{D} P}{\mathrm{D} t} \equiv P_{t}+J(\Phi, P) \equiv P_{t}+\Phi_{x} P_{y}-\Phi_{y} P_{x}=0
$$

where $\Phi$ is the flow stream function. For classical two-dimensional flow $P$ is just the ordinary vorticity $\nabla^{2} \Phi$; on the beta-plane, $P=\nabla^{2} \Phi+\beta y$. It is easily checked that the spatial integral of energy $\frac{1}{2}|\nabla \Phi|^{2}$ is conserved in both cases. Obviously the spatial integral of any function of $P$ is likewise conserved. In the case of ordinary two-dimensional flow, the spatial integral of $\frac{1}{2} P^{2}$ is just the integrated enstrophy, which is quadratic. On the beta-plane, by contrast,

$$
\iint \frac{1}{2} P^{2} \mathrm{~d} x \mathrm{~d} y=\iint \frac{1}{2}\left(\nabla^{2} \Phi\right)^{2} \mathrm{~d} x \mathrm{~d} y+\beta \iint y \nabla^{2} \Phi \mathrm{d} x \mathrm{~d} y+\frac{1}{2} \beta^{2} \iint y^{2} \mathrm{~d} x \mathrm{~d} y .
$$

The last term of (3.2) is a constant of the geometry, and the penultimate is just $\beta$

$\dagger$ On the other hand, such development appears to be suppressed by even moderate values of $\beta$ (Holloway 1984). 
times Kelvin's impulse, which is separately conserved (with or without $\beta$ ) in the zonally-symmetric problem considered here. The integrated enstrophy is thus a quadratic invariant both of classical two-dimensional flow, and of two-dimensional flow on a beta-plane. $\dagger$ It follows that the effect of the beta-plane geometry, allowing the existence of Rossby waves, is expressed only in the higher-order invariants of the problem. Therefore it is important to determine whether these invariants constrain the flow evolution in a significant manner.

As was pointed out in $\$ 1$, the fact that $\beta$ does not alter the quadratic invariants means that, if the dynamics is ergodic both with and without $\beta$, then the flow statistics in the two cases should, eventually, both become statistically isotropic. This is reflected in the fact that the absolute equilibrium solutions for an inviscid truncated representation are independent of $\beta$ (Salmon et al. 1976). The disequilibrium behaviour is, on the other hand, known to be affected by $\beta$ (Rhines 1975; Holloway \& Hendershott 1977): in unforced evolution the nonlinear cascades slow down, the reverse energy cascade apparently arresting at a scale $L_{\beta} \approx(U / \beta)^{\frac{1}{2}}$, the wave steepness characterizing the large-scale motion decreases, and the flow develops a strong tendency to zonal anisotropy. It has nevertheless been plausibly argued (e.g. Holloway 1986) that this behaviour just reflects an increase in the timescale characterizing the approach to isotropy.

\section{The finite-amplitude generalized Rayleigh theorem}

The proof of non-ergodicity given in this paper relies on the existence of a finite-amplitude generalized Rayleigh theorem, stated as follows: disturbances to a steady zonal basic state with a non-vanishing absolute-vorticity gradient $Q_{y}$ have their (disturbance) enstrophy at any time $t$ bounded in terms of their initial enstrophy at $t=0$ according to

$$
\iint q^{2}(x, y, t) \mathrm{d} x \mathrm{~d} y \leqslant \frac{\left|Q_{y}\right|_{\max }}{\left|Q_{y}\right|_{\min }} \iint q^{2}(x, y, 0) \mathrm{d} x \mathrm{~d} y .
$$

Here $q$ is the disturbance vorticity, namely the total absolute vorticity less $Q$. The corresponding statement in the spherical case is (A 2).

The bound (4.1) appears to have been stated first by McIntyre \& Shepherd (1987, equation (6.28)), where it was derived by a limiting process from a version of one of Arnol'd's stability theorems for non-parallel steady flow. It can also be inferred from the finite-amplitude 'generalized Eliassen-Palm' theorem presented in Killworth \& McIntyre (1985). But the direct proof of (4.1) is so straightforward that, for the sake of completeness, it is worth presenting here.

The governing equation is (3.1). Now consider an $x$-invariant 'basic state' $\Phi=\Psi, P=Q$, with monotonic $Q(y)$; since the velocity associated with $\Psi$ must be purely zonal, it follows that the advection term $J(\Psi, Q)$ vanishes and $\Psi, Q$ is itself a steady solution to (3.1). Defining the 'disturbance' $\psi, q$ in the natural way

$$
\Phi \equiv \Psi+\psi, \quad P \equiv Q+q,
$$

with $q \equiv \nabla^{2} \psi$, the governing equation (3.1) may then be written in the form

$$
\frac{\mathrm{D} q}{\mathrm{D} t}=-J(\psi, Q)=-\psi_{x} Q_{y}=-\frac{\mathrm{D} Q}{\mathrm{D} t} .
$$

† In a bounded beta-plane geometry, of course, conservation of Kelvin's impulse would be lost because of the broken zonal symmetry, and so the enstrophy would not be a conserved quantity. 
The $x$-invariance of the basic state $Q(y)$ allows the definition of the inverse function $Y_{0}(Q)$. Now introduce the second-order disturbance quantity

$$
A(Q, q) \equiv-\int_{0}^{q}\left\{Y_{0}(Q+\tilde{q})-Y_{0}(Q)\right\} \mathrm{d} \tilde{q}
$$

Note that in the limit of small-amplitude disturbances,

$$
A(Q, q) \approx-\frac{1}{2} Y_{0}^{\prime}(Q) q^{2}=-\frac{1}{2} \frac{q^{2}}{Q_{y}}
$$

$A(Q, q)$ is of definite sign whenever $Q(y)$, and thus $Y_{0}(Q)$, are monotonic, as assumed here. It may be verified that

$$
\frac{\partial A}{\partial Q}=-Y_{0}(Q+q)+Y_{0}(Q)+q Y_{0}^{\prime}(Q)
$$

and

and thence, using (4.3), that

$$
\frac{\partial A}{\partial q}=-Y_{0}(Q+q)+Y_{0}(Q)
$$

$$
\frac{\mathrm{D} A}{\mathrm{D} t}=q Y_{0}^{\prime}(Q) \psi_{x} Q_{y}=\left(\psi_{x x}+\psi_{y y}\right) \psi_{x}=\frac{1}{2}\left(\left(\psi_{x}\right)^{2}-\left(\psi_{y}\right)^{2}\right)_{x}+\left(\psi_{x} \psi_{y}\right)_{y}
$$

When averaged in $x$, this is just the generalized Eliassen-Palm theorem of Killworth \& McIntyre (1985, equation (5.17)). Integration of (4.6) over a suitable domain (such that the flux terms vanish) then yields the global conservation law

$$
\frac{\mathrm{d}}{\mathrm{d} t} \iint A(Q, q) \mathrm{d} x \mathrm{~d} y=0 .
$$

One may consider $A$ to be the density of a pseudomomentum. The way in which the conservation law (4.7) is related to the symmetry properties of the basic state is discussed by McIntyre \& Shepherd (1987, §7).

To derive (4.1) from (4.7), it suffices to note the inequalities

$$
\frac{\frac{1}{2} q^{2}}{\left|Q_{y}\right|_{\max }}=\int_{0}^{Q}\left|Y_{0}^{\prime}(Q)\right|_{\min } \tilde{q} \mathrm{~d} \tilde{q} \leqslant|A(Q, q)| \leqslant \int_{0}^{q}\left|Y_{0}^{\prime}(Q)\right|_{\max } \tilde{q} \mathrm{~d} \tilde{q}=\frac{\frac{1}{2} q^{2}}{\left|Q_{y}\right|_{\min }},
$$

the min and the max being taken over all values of $Q$ in the entire flow. A similar result was used by Arnol'd (1966) in the context of non-parallel flow to derive his nonlinear stability theorems. The bound (4.1) follows immediately from (4.8).

\section{A demonstration of non-ergodicity for nonlinear Rossby waves}

Attention is now restricted to Rossby waves on a beta-plane, for which $P=\nabla^{2} \Phi+\beta y$. The discussion of $\S 1$ established that, to disprove ergodic dynamics on the phase-space surface of constant energy and enstrophy, it is sufficient to demonstrate that the flow cannot become isotropic from a certain non-trivial set of initial conditions. (Obviously it is not necessary to demonstrate this in order to disprove ergodicity; focusing on isotropy is merely a convenient way of showing that a trajectory cannot access all of phase space.) It is easy to see heuristically why (4.1) can be used for this purpose: isotropy requires a large-amplitude departure from zonally-symmetric motion, but (4.1) bounds the extent of such a departure, provided the zonally-symmetric motion is weak enough to be stable. A rigorous proof follows. 
It is notationally convenient to consider doubly-periodic geometry, although the proof for an infinite beta-plane would be essentially the same. If the stream function is doubly periodic then the two components of linear momentum are identically zero. Any (smooth) flow can then be decomposed into a discrete (though infinite) sum of Fourier modes,

$$
\Phi(x, y, t)=\sum_{k, l} \hat{\phi}_{k l}(t) \exp \{\mathrm{i}(k x+l y)\}
$$

(real part understood), which are energetically orthogonal. The problem can be scaled so that the domain size is $2 \pi$ square, and thus only integer $k, l$ need be considered.

For the purpose of illustration, consider first the following simple example of a basic flow

$$
U(y)=U_{0} \cos (m y), \quad Q(y)=\beta y+U_{0} m \sin (m y),
$$

with $U_{0}$ and $m$ positive constants, and with $\beta>U_{0} m^{2}$ so that $Q(y)$ is monotonic. Then

$$
\left|Q_{y}\right|_{\max }=\beta+U_{0} m^{2}, \quad\left|Q_{y}\right|_{\min }=\beta-U_{0} m^{2} .
$$

Suppose that at the initial time, $t=0$, the disturbance does not project onto the basic flow; that is, the $(k, l)=(0, \pm m)$ Fourier modes of the total flow consist just of the basic flow. Then the disturbance enstrophy at $t=0$ is given by

$$
z_{0} \equiv \frac{1}{4 \pi^{2}} \iint \frac{1}{2} q^{2}(x, y, 0) \mathrm{d} x \mathrm{~d} y=\sum_{(k, l) \neq(0, \pm m)} \frac{1}{2}\left(k^{2}+l^{2}\right)^{2}\left|\hat{\phi}_{k l}(0)\right|^{2} .
$$

The finite-amplitude generalized Rayleigh theorem (4.1) thus takes the form

$$
\frac{1}{4 \pi^{2}} \iint \frac{1}{2} q^{2}(x, y, t) \mathrm{d} x \mathrm{~d} y \leqslant\left\{\frac{\beta+U_{0} m^{2}}{\beta-U_{0} m^{2}}\right\} z_{0} .
$$

Now, the enstrophy of the total flow, which is a conserved quantity, is just

$$
Z \equiv \frac{1}{4 \pi^{2}} \iint \frac{1}{2}\left\{\nabla^{2} \Phi(x, y, 0)\right\}^{2} \mathrm{~d} x \mathrm{~d} y=\frac{1}{4} U_{0}^{2} m^{2}+z_{0}
$$

If one assumes a state of isotropy at some future time $t$, then the amount of enstrophy in zonally-asymmetric $(k \neq 0)$ modes must at the very least (and this is a weak bound indeed) be one-half of the enstrophy of the total flow. That is, isotropy requires

$$
\frac{1}{2} Z \leqslant \sum_{\substack{k, l \\(k \neq 0)}} \frac{1}{2}\left(k^{2}+l^{2}\right)^{2}\left|\hat{\phi}_{k c l}(t)\right|^{2} \leqslant \frac{1}{4 \pi^{2}} \iint \frac{1}{2} q^{2}(x, y, t) \mathrm{d} x \mathrm{~d} y .
$$

A contradiction between (5.4) and (5.6) is thus obtained if the right-hand side of (5.4) is less than the left-hand side of (5.6), viz. (using (5.5))

$$
\begin{aligned}
2\left(\beta+U_{0} m^{2}\right) z_{0}<\left(\beta-U_{0} m^{2}\right) Z=\left(\beta-U_{0} m^{2}\right) & \left(\frac{1}{4} U_{0}^{2} m^{2}+z_{0}\right) \\
& \Leftrightarrow z_{0}<\left\{\frac{\beta-U_{0} m^{2}}{\beta+3 U_{0} m^{2}}\right\}_{4} U_{0}^{2} m^{2} .
\end{aligned}
$$

This simple example is perhaps sufficient to make the point. More generally, given some initial condition

$$
\Phi(x, y, 0)=\sum_{k, l} \hat{\phi}_{k l}(0) \exp \{i(k x+l y)\}
$$

and thus specified values of total energy and enstrophy, choose a 'basic state' consisting of a finite number of the zonally-symmetric $(k=0)$ modes, viz.

$$
\Psi(x, y)=\sum_{|l|=l_{1}}^{l_{2}} \hat{\phi}_{0 l}(0) \exp (\mathrm{i} l y)
$$


It is convenient to denote the restricted summation in (5.8) by $\Sigma_{l}^{\prime}$. In order to obtain a non-vanishing basic-state absolute-vorticity gradient, the range of $l$ in (5.8) must be chosen such that

Then

$$
\gamma \equiv \Sigma_{l}^{\prime}|l|^{3}\left|\hat{\phi}_{0 \ell}(0)\right|<\beta
$$

As before, the basic state has been chosen so that the disturbance does not project onto it at $t=0$. The same manipulations therefore go through, and one obtains a contradiction between isotropy and the finite-amplitude bound (4.1) if the initial disturbance enstrophy $z_{0}$ satisfies

$$
z_{0}<\left\{\frac{\beta-\gamma}{\beta+3 \gamma}\right\} \Sigma_{l}^{\prime} l_{2}^{4}\left|\hat{\phi}_{0 l}(0)\right|^{2}
$$

which is a more general version of (5.7). To complete the proof of non-ergodicity, it is necessary to show that the set of initial conditions from which the flow cannot become isotropic, namely that consistent with (5.11) (subject to (5.9)), is non-trivial in the sense of having positive measure. This is done in Appendix B.

It will be apparent that taking (5.6) as a consequence of isotropy is in fact a rather weak bound. In particular cases one could perhaps make a statement that isotropy required instead

$$
\frac{1}{r} Z \leqslant \sum_{\substack{k, l \\(k \neq 0)}} \frac{1}{2}\left(k^{2}+l^{2}\right)^{2}\left|\hat{\phi}_{k l}(t)\right|^{2}
$$

for some $r \in(1,2]$, the exact value depending on the circumstances. (In the special case of (5.1), for instance, one might use $r=(m+1) / m$, taking advantage of the fact that the fraction of enstrophy in zonally-symmetric modes would be at most $1 /(m+1)$ if the flow were isotropic.) Using (5.12) in place of (5.6) then yields a contradiction if

$$
z_{0}<\left\{\frac{\beta-\gamma}{(r-1) \beta+(r+1) \gamma}\right\} \Sigma_{l}^{\prime}{ }_{2}^{1} l^{4}\left|\hat{\phi}_{0 l}(0)\right|^{2}
$$

Note that for $1<r<2,(5.13)$ is easier to satisfy (i.e. it allows larger $z_{0}$ ) for a given basic state than is (5.11); this is to be expected, since (5.12) is a tighter bound than (5.6).

\section{Estimation of the extent of non-ergodic behaviour}

The calculations of $\$ 5$ show that, whenever a basic flow and disturbance can be defined satisfying (5.9) and (5.11), the dynamics cannot be ergodic on the phase-space surface corresponding to the same values of (total) energy and enstrophy. This is because, for any given initial configuration, there is then a finite (i.e. non-trivial) portion of that phase-space surface which cannot be entered. Therefore, to exclude ergodic behaviour for any given values of energy and enstrophy, it is sufficient to find some basic flow and disturbance, consistent with those values, for which (5.9) and (5.11) hold. In this section an attempt is made to estimate the values of $\beta$ (for given energy and enstrophy) for which this can be done.

One is at liberty to scale length and time in any convenient fashion. Length has already been scaled (by $L / 2 \pi$ ) so that the doubly-periodic domain is $2 \pi$ square, and wavenumbers are integers; now time is scaled so that the total energy of the flow (which is invariant) is unity, and the r.m.s. velocity $U$ is therefore $\sqrt{ } 2$. With this 
scaling, the only free parameters are dimensionless enstrophy, $Z^{\prime}=Z L^{2} / 2 \pi^{2} U^{2}$, and dimensionless beta, $\beta^{\prime}=\beta L^{2} / 2 \sqrt{ } 2 \pi^{2} U$; their ratio is just one-half the average wave steepness $\epsilon=2 \sqrt{ } 2 Z / \beta U$, which is a measure of typical relative-vorticity gradients against the planetary-vorticity gradient $\beta$. Note that this scaling implies $Z^{\prime} \geqslant 1$. For convenience, the primes are now dropped and reference is made only to dimensionless quantities.

The problem thus reduces to that of finding, for given $\beta$ and $Z$, some basic flow such that (5.9) and (5.11) hold. Now (5.9) requires a sufficiently weak relative-vorticity gradient in the basic flow, and this gradient is evidently minimized, for given basic-flow enstrophy, by choosing a basic flow of only a single scale, viz. (5.1). If the basic-flow energy is chosen to be $\alpha^{2} \leqslant 1$, then the basic-flow enstrophy is just $\alpha^{2} m^{2}$, $U_{0}$ in (5.1) equals $2 \alpha$, and the maximum relative-vorticity gradient, $\gamma$, is given by $\gamma=2 \alpha m^{2}$. Choosing the disturbance to be energetically orthogonal to this specially contrived basic flow, so that $z_{0}=Z-\alpha^{2} m^{2}$, condition (5.9) amounts to

$$
\gamma=2 \alpha m^{2}<\beta
$$

while (5.11) takes the form

$$
\begin{gathered}
Z-\alpha^{2} m^{2}<\left\{\frac{\beta-2 \alpha m^{2}}{\beta+6 \alpha m^{2}}\right\} \alpha^{2} m^{2} \\
\Leftrightarrow 4 \alpha^{3}\left(m^{2}\right)^{2}+\left(2 \beta \alpha^{2}-6 Z \alpha\right) m^{2}-\beta Z>0 .
\end{gathered}
$$

To prove non-ergodicity for given $\beta$ and $Z$, it is sufficient to be able to choose some (integer) $m$ such that (6.1) and (6.3) hold. By considering the positive root for $m^{2}$ of (6.3), it can be seen that this choice is possible provided that

$$
\frac{\alpha m^{2}}{\beta} \leqslant \frac{Z}{\beta \alpha}<\frac{1}{2}
$$

the left inequality following from $\alpha^{2} m^{2} \leqslant Z$. To obtain the widest range of wave steepness $\epsilon=2 Z / \beta$ from (6.4), one must evidently try to maximize $\alpha$. (This amounts to putting as much of the total energy and enstrophy as possible into the basic flow.) If $Z$ happens to be the square of an integer, then one may choose $\alpha=1$ and $Z=m^{2}$, so that non-ergodicity is proved for $\epsilon<1$. More generally, $\alpha$ is maximized by choosing $m$ to be the first integer greater than (or equal to) $Z^{\frac{1}{2}}$, viz.

$$
Z^{\frac{1}{2}} \leqslant m<Z^{\frac{1}{2}}+1
$$

and by taking all the disturbance energy to be at unit wavenumber, so that $Z=\alpha^{2} m^{2}+\left(1-\alpha^{2}\right)$. Thus

$$
\alpha^{2}=\frac{Z-1}{m^{2}-1}>\frac{Z-1}{Z+2 Z^{\frac{1}{2}}},
$$

the inequality being obtained by using (6.5). Finally, it may be seen from (6.6) that (6.4) is guaranteed to hold, and non-ergodicity proven, if

$$
\beta>2 Z\left\{\frac{Z+2 Z^{\frac{1}{2}}}{Z-1}\right\}^{\frac{1}{2}} \equiv 2 Z f(Z) .
$$

$f(Z)$ is a dimensionless function of the scaled enstrophy (the latter being just the second moment of the energy spectrum) and takes positive values between unity and infinity. In the limit of large $Z$ (which corresponds to a situation where the domain size is much larger than the size of a typical eddy), $f(Z)$ approaches unity and the condition $\epsilon<1$ for non-ergodicity is again obtained. 
Of course the above calculations only determine a sufficient condition on $\beta$ (given $Z$ ) for non-ergodicity. It is possible that a more clever choice of basic flow and disturbance, or a more efficient use of inequalities, would produce a less restrictive condition. However, attempts to use the apparently more powerful condition (5.13) in place of (5.11) have not provided an improvement on the result (6.7).

\section{Discussion}

This paper addresses the question of whether the dynamics of inviscid twodimensional flow on a beta-plane, or on the surface of a rotating sphere, is ergodic on the phase-space surface of constant energy and enstrophy (these being the two quadratic invariants of the problem). Ergodicity in this sense cannot generally be proven for fluid systems, but has commonly been hypothesized to hold for classical two-dimensional flow (e.g. Kraichnan 1975). Its relevance for nonlinear Rossby waves, which possess the same quadratic invariants as classical two-dimensional flow, has been the subject of much debate (e.g. Salmon 1982; Bennett \& Haidvogel 1983; Holloway 1986). If Rossby-wave dynamics were ergodic in this sense it would mean that two-dimensional flow on a beta-plane must eventually become isotropic in the limit of large time, a tendency for which there is no known numerical support.

It has been shown here that, for sufficiently strong $\beta$, two-dimensional flow on a beta-plane is not in fact ergodic in the sense described above; on the contrary, under these conditions it is impossible for any flow realization to sample the entire phase-space surface of constant energy and enstrophy. A corresponding result holds for flow on the surface of a rotating sphere, for a sufficiently rapid rotation rate $\Omega$ (see Appendix A). The specific result for the beta-plane is that, for dimensionless $\beta$ greater than a function of dimensionless enstrophy, i.e. (6.7), there are finite regions of the phase-space surface for which the flow is not isotropic, and from which trajectories cannot escape. Since the dynamical equations are time reversible, this implies conversely that for any given initial configuration satisfying $(6.7)$ there exists a finite region of the phase-space surface which cannot be entered. In the limit of dimensionless enstrophy much greater than unity, equivalently when the domain size is much larger than the size of a typical eddy (which is the regime of practical interest), (6.7) simplifies to a condition that the wave steepness $\epsilon=2 \sqrt{ } 2 Z / \beta U<1$. A similar result holds in the spherical case, but with $\epsilon=2^{\frac{1}{a}} a^{\frac{b}{2}} Z^{\frac{3}{3}} / \Omega U^{\frac{5}{2}}$ (see Appendix A).

This result is a direct consequence of a finite-amplitude Liapunov stability theorem for zonally symmetric flows with a non-vanishing absolute-vorticity gradient. The theorem (4.1) is derived from an exact conservation law (4.7) which itself relies on the material conservation of absolute vorticity. Thus it can be said that the proof of non-ergodicity given here has shown explicitly how the higher-order (nonquadratic) flow invariants play a constraining role in the flow evolution. It is believed that this is the only such demonstration of a rigorous nature.

One of the previous clues that ergodicity might not hold for nonlinear Rossby waves was the disinclination of numerical simulations of unforced beta-plane turbulence (e.g. Rhines 1975) to become isotropic in the limit of long time. Within the context of strictly inviscid dynamics the result of this paper can say nothing about the eventual outcome of such simulations; not only are the models truncated and (weakly) dissipative, but they are generally initialized in the strong-wave regime with $\epsilon>1$. However, the robust characteristic of these modelled flows is that a state is eventually reached where the wave steepness characterizing the large-scale flow is order unity or less (see Rhines 1975, figure 4). This change is accomplished by an 
irreversible cascade of enstrophy to very small scales (parameterized in the numerical simulations by a dissipation of enstrophy near the truncation limit), thereby decreasing both $Z$ and the effective $\epsilon$ at large scales. (Since the energy is essentially unchanged, the dimensionless scaling does not alter during the course of the simulation.) The definition of wave steepness used by Rhines is equal to $\sqrt{ } 2 /(1+B)$ times the wave steepness used here, where $B$ is a 'spectral breadth' parameter which for most of Rhines's experiments is much less than one. Since the dimensionless enstrophy $Z L^{2} / 2 \pi^{2} U^{2}$ is large in the experiments, this means that non-ergodicity is provable (only, of course, in the untruncated system described by (3.1)!) when Rhines's wave steepness is less than about $\sqrt{ } 2$. As can be seen from his table 1 and figure 4, some of Rhines's experiments do indeed cross this threshold during their evolution.

One cannot, of course, make a direct inference about the numerical simulations from this strictly inviscid theory; but it can perhaps be argued that in the ideal case of (3.1), the large-scale flow can become non-ergodic in a certain sense once the effective large-scale wave steepness becomes sufficiently small. In fact, since ergodic dynamics generally leads to spectral broadening, an enstrophy cascade, and a consequent decrease in the effective wave steepness, it might be argued that perpetually ergodic behaviour in unforced flow is an impossibility. One must raise the caveat that it is difficult to justify rigorously this notion of an effective large-scale wave steepness for two-dimensional flow, because the nonlinear interactions are not spectrally local; nevertheless the clear development of large-scale Rossby-wave propagation out of beta-plane turbulence (Rhines 1975) suggests that the idea is essentially correct. Therefore, the result of this paper gives quantitative support for Salmon's (1982, p. 75) conjecture that, beyond a certain time when the 'wave regime' is entered, the large-scale dynamics of beta-plane turbulence ceases to be ergodic.

The author would like to thank D. G. Dritschel, P. H. Haynes, M. N. Juckes, M. E. MeIntyre, R. Salmon, and especially R. L. Panetta, for helpful comments and discussions. The extension of the analysis to the spherical case was done following the suggestion of a referee. Support is acknowledged from the Natural Sciences and Engineering Research Council of Canada, the UK Natural Environment Research Council, and St Catharine's College, Cambridge.

\section{Appendix A. The case of a rotating sphere}

The preceding analysis is now repeated for the case of two-dimensional flow on the surface of a rotating sphere. The governing equation (3.1) then takes the form

$$
\frac{\mathrm{D} P}{\mathrm{D} t} \equiv P_{t}+J(\Phi, P) \equiv P_{t}+a^{-2} \Phi_{\lambda} P_{\mu}-a^{-2} \Phi_{\mu} P_{\lambda}=0,
$$

where $a$ is the radius of the sphere, $\mu \equiv \sin \phi, \phi$ is latitude, $\lambda$ is longitude, and $P=\nabla^{2} \Phi+2 \Omega \mu$ is the absolute vorticity. As in the planar case, the quadratic invariants are energy and enstrophy; the latter differs from $\frac{1}{2} P^{2}$ by a term involving Kelvin's impulse, or zonal angular momentum, which is separately conserved. It may be verified that the analysis of $\$ 4$ goes through in a straightforward manner, the exact invariant being (4.4) with $\mu_{0}$ replacing $Y_{0}$, and yields in place of (4.1) the bound

$$
\iint q^{2}(\mu, \lambda, t) \mathrm{d} \mu \mathrm{d} \lambda \leqslant \frac{\left|Q_{\mu}\right|_{\max }}{\left|Q_{\mu}\right|_{\min }} \iint q^{2}(\mu, \lambda, 0) \mathrm{d} \mu \mathrm{d} \lambda .
$$


Here the integrals are taken over the surface of the sphere, so $\mu$ goes from -1 to +1 and $\lambda$ from 0 to $2 \pi$. The disturbance vorticity has the explicit spherical-coordinate form

$$
q \equiv \nabla^{2} \psi=a^{-2}\left(1-\mu^{2}\right)^{-1} \psi_{\lambda \lambda}+a^{-2}\left\{\left(1-\mu^{2}\right) \psi_{\mu}\right\}_{\mu}
$$

and the basic-state absolute-vorticity gradient may be written as

$$
Q_{\mu}=2 \Omega+a^{-2}\left\{\left(1-\mu^{2}\right) \Psi_{\mu}\right\}_{\mu \mu}
$$

It is obvious from (A 4) that axisymmetric basic states with non-vanishing absolutevorticity gradients can be constructed, as in $\$ 5$, and that (A 2 ) can then be used to prove non-ergodicity for given total energy and enstrophy, provided $\Omega$ is sufficiently large. We now attempt to make this statement quantitative.

As in $§ 6$, it is convenient to scale time such that the total energy of the flow is unity and the r.m.s. velocity $U$ is therefore $\sqrt{ } 2$; length is scaled by the sphere radius $a$. With this scaling, the free parameters are dimensionless enstrophy, $Z^{\prime}=2 Z a^{2} / U^{2}$, and dimensionless rotation rate, $\Omega^{\prime}=\sqrt{ } 2 \Omega a / U$. Note that this scaling implies $Z^{\prime} \geqslant 2$. Primes are now dropped and variables are dimensionless unless stated otherwise.

From §6, we may anticipate that the strongest result for non-ergodicity will be obtained by constructing basic-state flows of a single meridional spectral mode, in this case a single Legendre polynomial

$$
\Psi(\mu)=\hat{\Psi}_{0} P_{n}^{0}(\mu)
$$

with associated relative vorticity

$$
\nabla^{2} \Psi=-n(n+1) \hat{\Psi}_{0} P_{n}^{0}(\mu) .
$$

In the above $P_{n}^{0}(\mu)$ is the associated Legendre polynomial of degree $n$ and zeroth order, normalized so that

$$
\frac{1}{4 \pi} \iint P_{n}^{0} P_{m}^{0} \mathrm{~d} \mu \mathrm{d} \lambda=\delta_{n}^{m}
$$

The maximum relative-vorticity gradient for the basic flow is obtained at $\mu=1$, viz.

$$
\gamma=n(n+1)\left|\hat{\Psi}_{0}\right|\left|\frac{\mathrm{d} P_{n}^{0}(1)}{\mathrm{d} \mu}\right|=n(n+1)\left|\hat{\Psi}_{0}\right| \frac{1}{2} n(n+1)(2 n+1)^{\frac{1}{2}},
$$

using well-known properties of Legendre polynomials (see e.g. Boer 1983, $\$ 2 d$ ).

Again from $§ 6$, we anticipate that the strongest result is obtained by putting as much of the total energy and enstrophy as possible into the constructed basic flow. If the basic-flow energy is $\alpha^{2} \leqslant 1$, then the basic-flow enstrophy is just $n(n+1) \alpha^{2}$, and $\hat{\Psi}_{0}$ in (A 5) is $\sqrt{ } 2 \alpha\{n(n+1)\}^{-\frac{1}{2}}$ (recalling that the enstrophy is here normalized by the surface area of the sphere, $4 \pi$; cf. (5.5) in the planar case). The condition for a non-vanishing basic-state absolute-vorticity gradient, analogous to (5.9) and (6.1), is then

$$
\gamma=\alpha\left\{\frac{1}{2} n^{3}(n+1)^{3}(2 n+1)\right\}^{\frac{1}{2}}<2 \Omega,
$$

while the bound on the size of the hypothesized disturbance, analogous to (5.11) and $(6.2)$, is

$$
z_{0}=Z-n(n+1) \alpha^{2}<\left\{\frac{2 \Omega-\gamma}{2 \Omega+3 \gamma}\right\} n(n+1) \alpha^{2}
$$

Unfortunately the algebra is much more complicated than in the planar case, and it has not been possible to obtain an explicit expression like (6.4) which would guarantee satisfaction of both (A 9) and (A 10). But in the limit of large $Z$ (corre- 
sponding to a physical situation where the size of a typical eddy is much less than the sphere radius), the parameter $\alpha$ can be taken arbitrarily close to unity, and the condition for non-ergodicity collapses to (A 9) with $\alpha=1$, which is approximately

$$
\Omega>\frac{1}{2} n^{\frac{7}{2}} \approx \frac{1}{2} Z^{\frac{7}{4}}
$$

The result (A 11) is immediately expressible in terms of a condition on some average wave steepness $\epsilon$, provided that one takes $\epsilon$ to be a measure of the ratio of the relative to the planetary vorticity gradient in the region near the pole, $\mu \lesssim 1$, which is where the ratio is maximized under homogeneous conditions. From (A 9) and (A 11), a possible choice might be $\epsilon=Z^{\frac{7}{4}} / 2 \Omega$ (still using dimensionless variables), in which case (A 11) translates to $\epsilon<1$. In terms of dimensional variables this choice becomes $\epsilon=2^{\frac{1}{4}} \alpha^{\frac{5}{2}} Z^{2} / \Omega U^{5}$, which may look rather odd, but is presumably a better measure of wave steepness, because of the properties of spherical geometry, than the simpler choice $a Z / \Omega U$.

\section{Appendix B. Proof that the set of initial conditions consistent with (5.11) has positive measure}

It is sufficient to show that, for given values of the energy and enstrophy, and given $\beta$, an initial condition chosen at random has a finite (non-zero) chance of satisfying (5.11). The reason why this is not particularly obvious is that while (5.11) requires that a certain finite fraction of the enstrophy reside in $k=0$ modes, those modes do not represent a finite fraction of all the modes in the system. The first step is to show that the most likely initial condition has a finite fraction of its enstrophy in the $k=0$ modes. This most likely initial condition will have an isotropic energy spectrum $E(\kappa)$, where $\kappa^{2} \equiv k^{2}+l^{2}$. At each $\kappa$, the fraction of the enstrophy in the $k=0$ mode is (approximately) $1 / \pi \kappa$. Thus the fraction of the total enstrophy in $k=0$ modes, $\rho$ say, is given by

$$
\rho=\frac{1}{\pi Z} \int \kappa E(\kappa) \mathrm{d} \kappa
$$

A lower bound for the expression (B 1) is obtained by putting the enstrophy into the smallest scales possible: viz. with $E(\kappa)=a \kappa^{-(3+\nu)}$ for $\kappa \geqslant \kappa_{0}$, in the limit $\nu \rightarrow 0$. For this choice it is easily seen that $Z \sim a / \nu, U \sim a^{\frac{1}{2}} / \kappa_{0}$, and $\rho \sim \nu / \pi \kappa_{0}$. Under the further hypothesis that the wave steepness $\epsilon \equiv 2 \sqrt{ } 2 Z / \beta U<1$, it follows that

$$
\rho_{\min } \sim \frac{\nu}{\pi \kappa_{0}} \sim \frac{a}{\pi \kappa_{0} Z} \sim \frac{U^{2} \kappa_{0}}{\pi Z} \geqslant \frac{U^{2}}{\pi Z}>\frac{2 \sqrt{ } 2 U}{\pi \beta}
$$

and is finite. But if the most likely initial condition has a finite value of $\rho$, then there is certainly a finite probability that $\rho$ will be large enough to satisfy (5.11).

\section{REFERENCES}

ARnoL'D, V. I. 1966 On an a priori estimate in the theory of hydrodynamical stability. Izv. Vyssh. Uchebn. Zaved. Matematika 54, no. 5, 3-5. (English transl.: Am. Math. Soc. Transl., Series 2 79, 267-269 (1969).)

Babiano, A., Basdevant, C., Legras, B. \& Sadourny, R. 1984 Dynamiques comparées du tourbillon et d'un scalaire passif en turbulence bi-dimensionnelle incompressible. C.R.Acad. Sci. Paris 299, Série II, 601-604.

Basdevant, C. \& Sadourny, R. 1975 Ergodic properties of inviscid truncated models of two-dimensional incompressible flows. J. Fluid Mech. 69, 673-688.

Batchelor, G. K. 1953 The Theory of Homogeneous Turbulence. Cambridge University Press. 
Bennett, A. F. \& Haidvogel, D. B. 1983 Low-resolution numerical simulation of decaying two-dimensional turbulence. J. Atmos. Sci. 40, 738-748.

Botr, G. J. 1983 Homogeneous and isotropic turbulence on the sphere. J. Atmos. Sci. 40, 154-163.

Carnevale, G. F. 1982 Statistical features of the evolution of two-dimensional turbulence. J. Fluid Mech. 122, 143-153.

Carnevale, G. F., Frisch, U. \& Salmon, R. $1981 H$-theorems in statistical fluid dynamics. J. Phys. A 14, 1701-1718.

Fox, D. G. \& Orszat, S. A. 1973 Inviscid dynamics of two-dimensional turbulence. Phys. Fluids 16, 169-171.

Herring, J. R. 1975 Theory of two-dimensional anisotropic turbulence. J. Atmos. Sci. 32, 2254-2271.

Herrina, J. R. \& McWilliams, J. C. 1985 Comparison of direct numerical simulation of two-dimensional turbulence with two-point closure: the effects of intermittency. J. Fluid Mech. 153, 229-242.

Herring, J. R., Orszag, S. A., Kratchnan, R. \& Fox, D. G. 1974 Decay of two-dimensional homogeneous turbulence. J. Fluid Mech. 66, 417-444.

HollowaY, G. 1984 Contrary roles of planetary wave propagation in atmospheric predictability. In Predictability of Fluid Motions, Am. Inst. Phys. Conf. Proc. (ed. G. Holloway \& B. J. West), vol. 106, pp. 593-599.

Holloway, G. 1986 Eddies, waves, circulation, and mixing: statistical geofluid mechanics. Ann. Rev. Fluid Mech. 18, 91-147.

Holloway, G. \& Hendershott, M. C. 1977 Stochastic closure for nonlinear Rossby waves. J. Fluid Mech. 82, 747-765.

Ketcs, L. C. \& Orszaa, S. A. 1978 Randomness of low-order models of two-dimensional inviscid dynamics. Phys. Fluids 21, 162-168.

Kulworth, P. D. \& MoIntyre, M. E. 1985 Do Rossby-wave critical layers absorb, reflect or over-reflect? J. Fluid Mech. 161, 449-492.

Kratchnan, R. H. 1967 Inertial ranges in two-dimensional turbulence. Phys. Fluids 10, 1417-1423.

Kraichnan, R. H. 1975 Statistical dynamics of two-dimensional flow. J. Fluid Mech. 67, 155-175.

MoIntyre, M. E. \& Shepherd, T. G. 1987 An exact local conservation theorem for finiteamplitude disturbances to non-parallel shear flows, with remarks on Hamiltonian structure and on Arnol'd's stability theorems. J. Fluid Mech. 181, 527-565.

McWilliams, J. C. 1984 The emergence of isolated, coherent vortices in turbulent flow. J. Fluid Mech. 146, 21-43.

PeIerls, R. 1979 Surprises in Theoretical Physics. Princeton University Press.

Prigoane, I. 1980 From Being to Becoming. W. H. Freeman.

Rhines, P. B. 1975 Waves and turbulence on a beta-plane. J. Fluid Mech. 69, 417-443.

Salmon, R. 1982 Geostrophic turbulence. In Topics in Ocean Physics (ed. A. R. Osborne \& P. Malanotte-Rizzoli), vol. 80, pp. 30-78. Societa Italiana di Fisica, Bologna : North-Holland.

Salmon, R., Holloway, G. \& Hendershott, M. C. 1976 The equilibrium statistical mechanics of simple quasi-geostrophic models. J. Fluid Mech. 75, 691-703.

Tномpson, P. D. 1982 On the structure of the hydrodynamical equations for two-dimensional flows of an incompressible fluid: the role of integral invariance. In Mathematical Methods in Hydrodynamics and Integrability in Dynamical Systems, Am. Inst. Phys. Conf. Proc. (ed. M. Tabor \& Y. M. Treve), vol. 88, pp. 301-317. 